\title{
Dietary Effects on Blood Pressure and Incidence of Stroke in SHRSP
}

\section{(1) Effects of Various Protein Sources}

\author{
Kozo Oкамотo, Tsuneyuki Suzuki, Hiroyuki Ito, Nobuko Morita, \\ Hideo Mryake, Kazuo Yamamoto, and Tetsuo Murakami*
}

SHRSP separated by Okamoto, Yamori, and Nagaoka (Circulat Res 34-35 (suppl 1): 143, 1974) have reached the 18th generation of successive selective inbreeding $\left(\mathbf{F}_{42}\right)$, and have a continued high incidence (over $90 \%$ ) of spontaneous cerebrovascular lesions (stroke).

We have studied dietary effects upon blood pressure and incidence of stroke in SHRSP, and have reported them (Jap Heart J 17: 401, 1976. Presentation at the 2nd U.S.-Japan Seminar on SHR held in Newport Beach, April, 1976). We have obtained some more data after the previous report.

Materials and Methods:

SHRSP of the $F_{32}$ through the $F_{41}$ generation were used in this study. They were divided into 2 groups. The control group was fed the stock chow diet (Funabashi F-2). The experimental group was fed special diets prepared as follows: protein source $\mathrm{X} \%$ by weight, $\alpha$-corn starch $86-\mathrm{X} \%$, soy bean oil $5 \%$, salt mixture $4 \%$, vitamin mixture $1 \%$, and cellulose $4 \%$ from 5 to 6 weeks of age. As protein sources, 11 kinds of foods, that is white fish meal, casein, euphausia, etc, were used. Blood pressures were measured every week by the tail-pulse-pickup method without anesthesia. Body weights were recorded at the same time. Autopsies were performed soon after natural death and especially the brains were carefully examined in order to detect stroke lesions. Blood samples from each group were taken from the tail tip and examined biochemically and hematologically about serum total protein, serum cholesterol, serum triglycerides, and hematocrit.

\section{Results:}

1. In males, the increase of blood pressure was remarkably slower, significantly from 8 weeks through 30 weeks of age, in animals on the following diets: $50 \%$ white fish meal, 35\% euphausia without crust, 25\% euphausia without crust $10 \%$ white fish meal, $9 \%$ yolk $/ 25 \%$ egg white, $40 \%$ chlorella $/ 10 \%$ white fish meal, $40 \%$ hydrocarbon yeast $10 \%$ white fish meal, $40 \%$ sweet smelt, or $25 \%$ dried beef meal/ $25 \%$ meat bone meal, than it was in the control.

The diet containing $45 \%$ soy bean cake or $5 \%$ vegetable protein also markedly depressed the elevation of blood pressure, but the former showed moderate and the latter showed marked disturbances of growth.

2. In females, the diets containing $50 \%$ white fish meal or $25 \%$ euphausia without crust $/ 10 \%$ white fish meal showed a remarkable preventive effect upon the elevation of blood pressure.

From the Department of Pathology and Research Institute of Food Science, ${ }^{*}$ Kinki University School of Medicine, Osaka-fu. 
3. The diets containing $50 \%$ or $20 \%$ casein, $30 \%$ or $20 \%$ white fish meal, $50 \%$ dried beef meal (in males), $70 \%$ cottage cheese $19 \%$ soy bean cake or $30 \%$ squid diet showed moderate effects.

4. The diets containing $10 \%, 30 \%$ vegetable protein or $50 \%$ dried beef meal (in females) or the $40 \%$ spirulina $/ 10 \%$ white fish meal diet showed no or almost no effect.

5. The incidence of stroke and the average age of death were $89 \%$ (No. of rats with lesions/No. of examined rats $=187 / 211)$, and $243 \pm 102(\mathrm{M} \pm \mathrm{SE})$ days in control males and $83 \%(64 / 77), 357 \pm 75$ days in control females. Most of them have already died. On the other hand, most or some of SHRSP fed with the $50 \%$, $30 \%, 20 \%$ white fish meal or $50 \%, 20 \%$ casein diets are still alive. The incidence of stroke was $15 \%(7 / 46), 24 \%(5 / 21), 0 \%(0 / 12)$ in males respectively, and the average age of death was over 100 days higher than for the control. The results showed similar tendencies for females.

6. When SHRSP were fed with the $50 \%$ white fish meal diet or the $50 \%$ casein diet from the first feeding, the elevation of blood pressure was controlled, especially in the former, from an early age not only in males, but also in females. Though some of them are still alive, the incidence of stroke in both groups was $0 \%$ (0/24 white fish meal, $0 / 10$ casein) and the average age of death was $338 \pm 74.0$, $321 \pm 39.6$ days, respectively, in males. On the other hand, the $50 \%$ white fish meal was slightly effective for blood pressure, and effective for the prevention of stroke $(11 \%(1 / 9), 360 \pm 84.9$ days) when ingested from 10 weeks, but not effective at all when ingested from 15 weeks, or ingested from the first feeding and changed to stock chow diet from 5 weeks of age.

7. Serum cholesterol levels in groups fed with the $25 \%$ euphausia without crust $/ 10 \%$ white fish meal, $35 \%$ euphausia without crust, or $45 \%$ soy bean cake diet were $41 \pm 6.7,49 \pm 4.7,35 \pm 4.6 \mathrm{mg} / 100 \mathrm{ml}$, respectively, and were lower than the control SHRSP $(59 \pm 6.9 \mathrm{mg} / 100 \mathrm{ml})$. The other experimental groups were the same as the control. No significant differences were observed between the control group and the experimental groups in serum triglycerides, serum total protein and hematocrit levels.

\section{Summary with Discussion:}

The diet containing white fish meal, euphausia, euphausia/white fish meal, yolk/egg white, chlorella/white fish meal, hydrocarbon yeast/white fish meal, sweet smelt or dried beef meal/meat bone meal as a protein source could markedly depress the elevation of blood pressure, prevent the incidence of stroke and elongate the life-span in SHRSP. We observed marked or slight enlargements of the kidney (1.6-2.0 times as heavy as the control) and the liver (1.1-1.2 times) in SHRSP fed with the $50 \%$ white fish meal diet or the $50 \%$ casein diet over a long time, but did not observe it in feeding with the diet containing under about $30 \%$ crude protein. Therefore, the $35 \%$ euphausia or $25 \%$ euphausia $10 \%$ white fish meal diet seems to be most effective in preventing stroke in SHRSP.

The incidence of stroke in SHRSP was prevented by providing these diets from an early age-the earlier the better.

The $50 \%$ dried beef meal diet was effective, but $25 \%$ dried beef meal $/ 25 \%$ meat bone meal diet was more effective in males. It seems that not only protein but minerals play a role in the prevention of stroke. 
This study was supported by Science and Technology Agency of Japanese Government, Ministry of Education, Japanese Society for the Promotion of Science, and National Heart and Lung Institute of NIH (USA) (HL 17754-02). 